\title{
THERMAL AND MECHANICAL CHARACTERIZATION AND CALIBRATION OF HEATED MICROCANTILEVERS
}

\author{
Jungchul Lee, Thomas Beechem, Keunhan Park, Zhuomin Zhang, Samuel Graham, and William P. King \\ Woodruff School of Mechanical Engineering, Georiga Institute of Technology \\ Atlanta, GA USA
}

\begin{abstract}
This paper reports the thermal and mechanical characterization and calibration of heated atomic force microscope (AFM) cantilevers. The cantilevers are characterized during steady-state, pulsed, and periodic heating. Using a laser Raman technique, it is possible to measure local temperature and temperature distribution in the silicon cantilever with a resolution of $1{ }^{\circ} \mathrm{C}$ and $1 \mu \mathrm{m}$ while providing local surface stress measurements. This work provides improved understanding of thermal, electrical, and mechanical behaviors of silicon heated AFM cantilevers thereby enabling new applications for the device.
\end{abstract}

\section{INTRODUCTION}

Heated microcantilevers were originally developed for thermomechanical data storage $[\mathbf{1 , 2}]$ however these cantilevers are additionally useful for highly sensitive metrology [3], nanometerscale manufacturing [4], and sensing heat flows $<1 \mathrm{nW}$. These applications beyond data storage demand strict requirements for device characterization and in particular temperature calibration well beyond what is required for data storage.

This paper reports advancements in characterization and calibration of these heated microcantilevers with temperature precision of $5{ }^{\circ} \mathrm{C}$ between room temperature and $800{ }^{\circ} \mathrm{C}$, in both time domain and frequency domain operation from steady-state to $>1 \mathrm{MHz}$. For this research, we have developed a novel laser Raman technique that can simultaneously measure temperature and intrinsic stress in the cantilever with $1 \mu \mathrm{m}$ resolution.

\section{MICROFABRICATED CANTILEVERS}

The silicon microcantilever-heaters were fabricated in our group at the Georgia Institute of Technology using a standard silicon-on-insulator process for cantilever fabrication. The fabrication processes were adopted from previously published reports on heated microcantilever fabrication [1] but modified to accommodate our microfabrication facility. The cantilever tip was formed using an oxidation sharpening process [5] and had a radius of curvature near $20 \mathrm{~nm}$. The cantilever was made electrically active through two phosphorous doping steps: Two parallel cantilever legs and heater region near the free end were doped to $1 \times 10^{20}$ and $1 \times 10^{17} \mathrm{~cm}^{-3}$, respectively. Therefore, the heater region was more highly resistive than the rest of the cantilever. Figure 1 shows scanning electron microscope (SEM) and infrared (IR) microscope images of a ' $U$ ' shape heated microcantilever, indicating atomically sharp tip, low intrinsic stress, and substantial heating only near the free end of the cantilever, respectively. The development of an all-silicon heated AFM cantilever was a significant advancement over previous work as silicon composed cantilevers having integrated heaters can reach higher temperatures, heat more quickly, and have reduced thermallyinduced bending. However, detailed investigations showed that thermally-induced bending was not entirely suppressed.
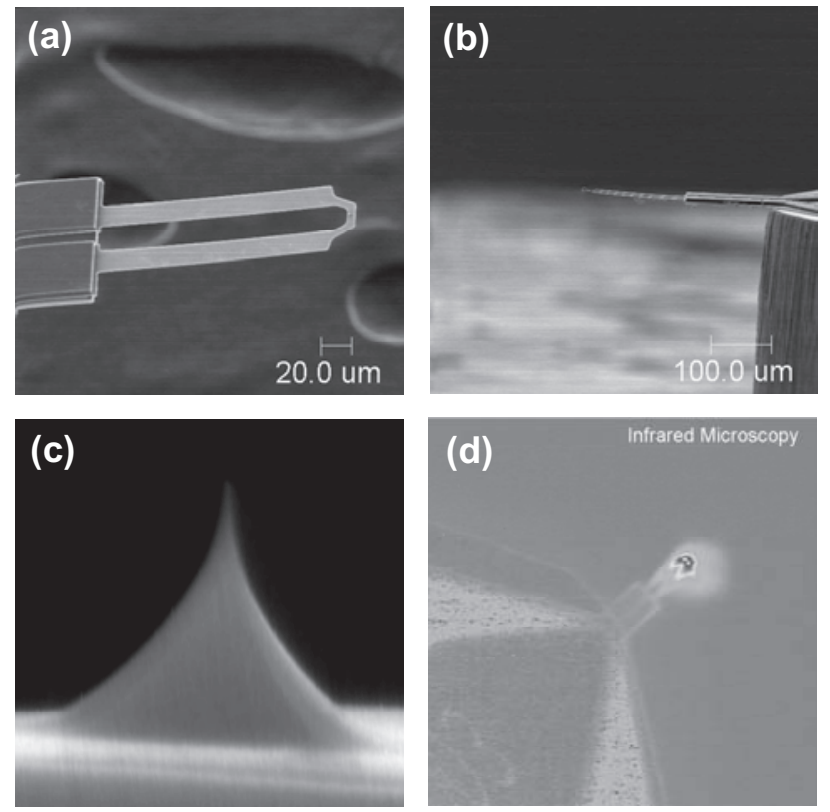

Figure 1. (a)-(c) SEM images of a microcantilever heater, side view showing low intrinsic stress, and cantilever tip. (d) IR microscope image of the heater cantilever during steady electrical excitation. The IR image is approximately $0.5 \mathrm{~mm}$ square. The doped silicon cantilever is fabricated in a ' $U$ ' shape such that it forms a continuous electrical path. The region near the cantilever free end is a highly resistive heater and the legs have lower electrical resistance such that they carry electricity. The IR image confirms substantial heating near only the free end of the cantilever.

For heated AFM cantilevers, the characterization of temperature and stress are important to assess the operational bounds of the device while ensuring reliability. In the operation of these devices, temperature measurements are often performed without regard for simultaneous stress evolution due to the difficulty in performing such measurements concurrently. In general, the measurement of temperature and stress in these devices is not trivial due to their size scale and the temperature range over which they operate.

\section{ELECTRICAL CHARACTERIZATION}

To characterize cantilever heating, the cantilever electrical response was examined with $\mathrm{DC}$, square pulse, and $\mathrm{AC}$ excitation; shown in Figs. 2-4. In both DC mode (Fig. 2a,b) and during short pulses (Fig. 2c,d), the cantilever exhibits highly nonlinear temperature coefficient of electrical resistance (TCR) and thermal runaway [6] where the TCR changes from positive to negative. The nonlinear behavior coupled with the cantilever thermal time constant near $100 \mu$ s significantly complicates the cantilever AC response, which depends upon both frequency and excitation voltage (Fig. 3). Low frequency oscilloscope traces in Fig. 3a-c 
show that the cantilever resistance is linear when the input voltage is low but becomes nonlinear as voltage is increased. All $I-V$ curves intersect with the origin, indicating that the electric power is totally dissipated by Joule heating. In Fig. 3d, voltage and current traces are plotted for $5 \mathrm{~V}$-rms and $1 \mathrm{MHz}$ as an example of high frequency response. The most apparent difference from the low frequency region is the large phase difference between the voltage and current. This phase difference suggests that the impedance plays an important role in the cantilever electric response.
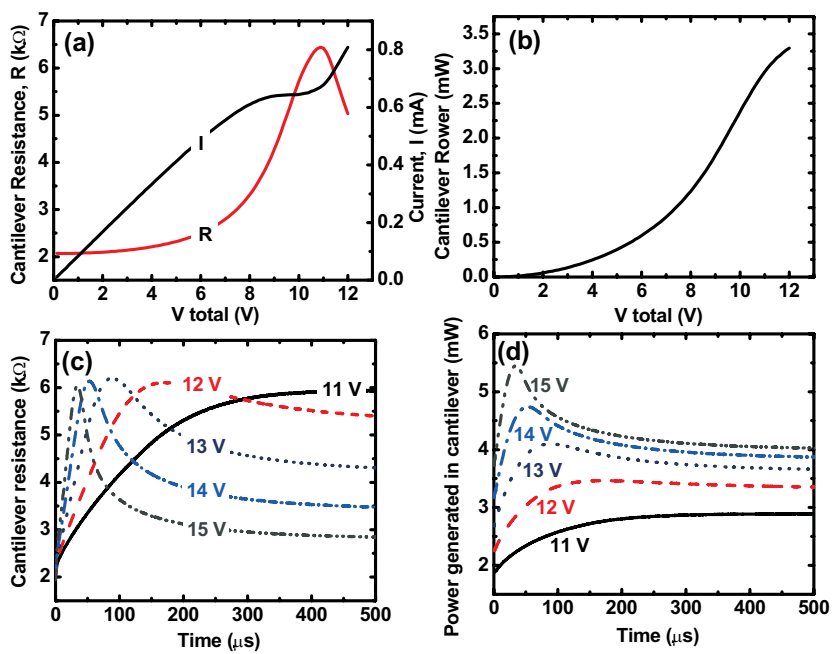

Figure 2. DC response of a heated AFM cantilever as a function of excitation voltage: (a) cantilever resistance and current (b) cantilever power. Square pulse response as a function of time at five different voltage settings: (c) Cantilever resistance (d) cantilever power.
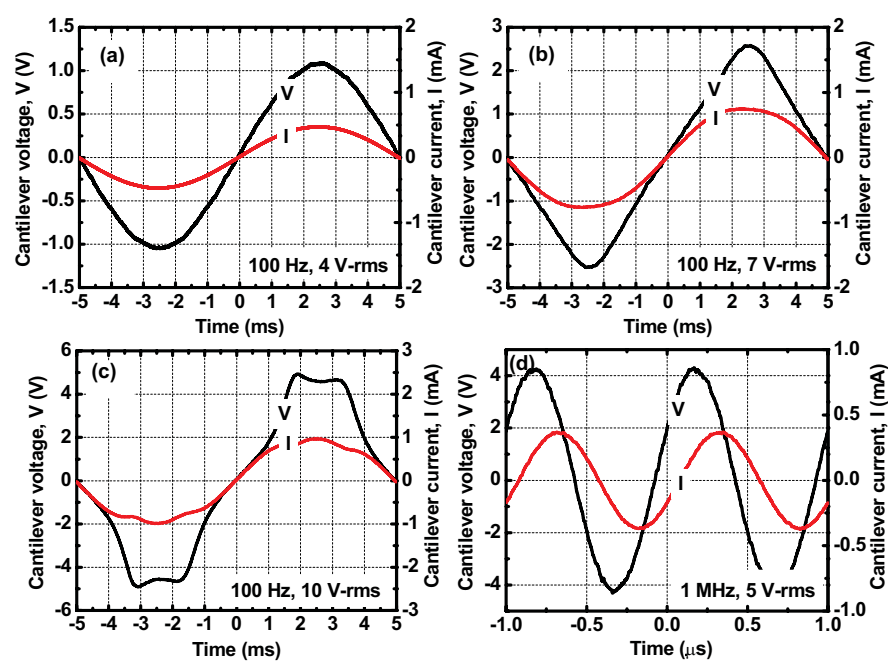

Figure 3. AC response of a heated AFM cantilever as a function of time at (a) low frequency and low voltage: $100 \mathrm{~Hz} \& 4 \mathrm{~V}$-rms (b) low frequency and intermediate voltage: $100 \mathrm{~Hz} \& 7 \mathrm{~V}$-rms (c) low frequency and high voltage: $100 \mathrm{~Hz} \& 10 \mathrm{~V}$-rms (d) high frequency: $1 \mathrm{MHz} \& 5 \mathrm{~V}$-rms.

Frequency domain characterization (Figs. 4,5) enables frequency modulated experiments which are desirable for measuring heat flows $<1 \mathrm{nW}$, as these experiments have increased signal to noise ratio and suppression of $1 / \mathrm{f}$ noise. When the heated

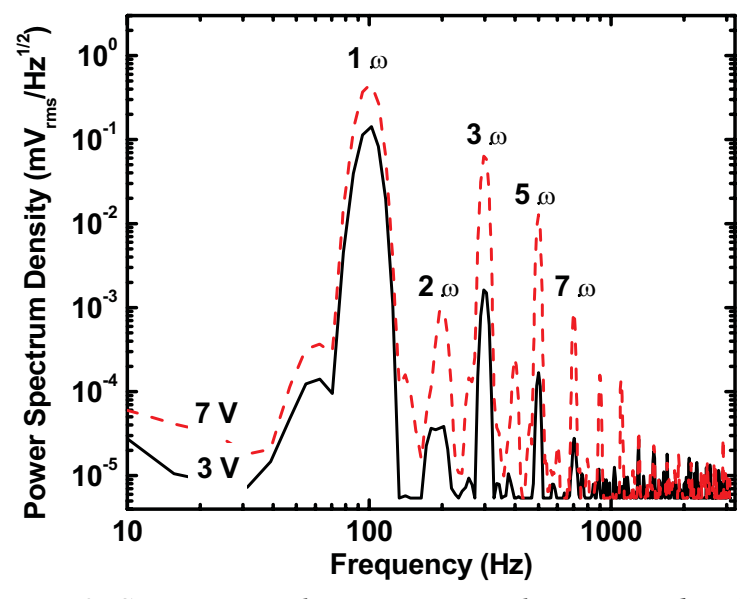

Figure 4. Spectrum analysis compares the magnitude of high order harmonics at two AC excitation voltages where the driving frequency is $100 \mathrm{~Hz}$. As excitation voltage increases, $3 \omega$ and $5 \omega$ components become significant such that they result in highly complex nonlinearity in the cantilever resistance.
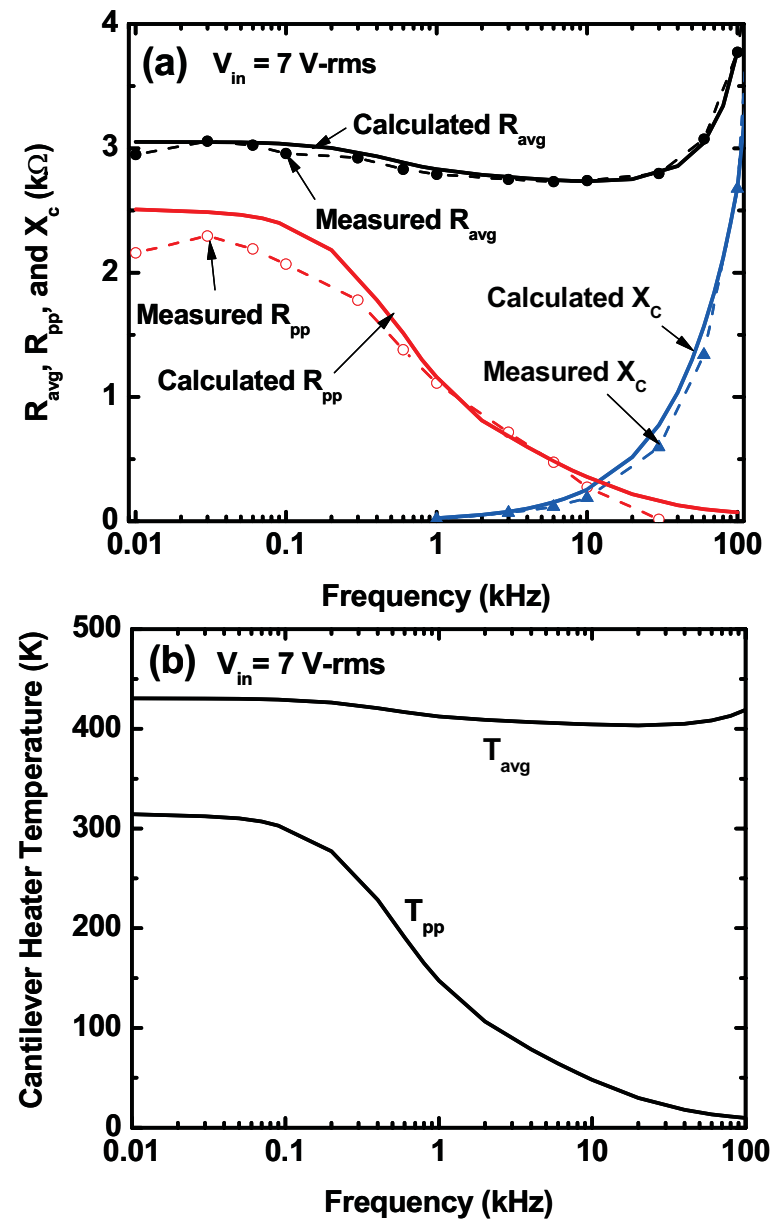

Figure 5. Transient heat transfer simulation was performed and results are compared with experiments. (a) comparison of the cantilever impedances that are experimentally obtained and simulated when excitation voltage is $7 \mathrm{~V}$-rms. $R_{\text {avg }}, R_{p p}$, and $X_{c}$ are average resistance, peak-to-peak resistance, and reactance, respectively. (b) Calculated cantilever heater temperature for the same case as (a). $T_{a v g}$ and $T_{p p}$ are average and peak-to-peak heater temperature, respectively. 
cantilever is operated with oscillatory electrical excitation having frequency $\omega$, the power dissipation in the cantilever will oscillate at frequency $2 \omega$ and cantilever temperature/resistance will follow the power oscillation. The generated $2 \omega$ resistance will generate $3 \omega$ voltage oscillation in conjunction with $1 \omega$ current input and higher overtones will be cascaded in a similar fashion. Fig. 4 shows power spectrum density of the heated cantilever with 100 $\mathrm{Hz}$ AC excitation. As AC voltage increases, more high order overtones become detectable due to highly nonlinear cantilever resistance and thermal runaway. In general, the amplitude of these harmonic overtones increases with increasing driving voltage amplitude and decreasing driving frequency.

Combined measurements and 1D numerical modeling allow estimates of both steady and oscillatory heating in the cantilever (Fig. 5). Fig. 5a shows frequency-dependent cantilever resistance and reactance when the input voltage is fixed at $7 \mathrm{~V}$-rms. The calculated values match well with the experimental data: only the simulated peak-to-peak resistance overestimates the measured data by $7.4 \%$ at low frequencies. This deviation could be attributed to an underestimate of the volumetric heat capacity of the cantilever or the variation of the effective heat transfer coefficient. The cantilever heater temperature in Fig. $5 \mathrm{~b}$ shows a similar trend as the resistance. The peak-to-peak values of cantilever resistance and heater temperature maintain their magnitudes until around $100 \mathrm{~Hz}$ and then decrease to become negligibly small.

\section{MECHANICAL CHARACTERIZATION}

In AFM operation, the interaction between the cantilever tip and the underlying substrate is of great importance and requires precise knowledge of the mechanical properties such as spring constant and resonance frequency. For mechanical characterization, thermal noise spectrum of the heated cantilever was measured using an Asylum MFP-3D AFM. Resonance frequency and $\mathrm{Q}$ factor were directly obtained from the measured spectral noise density shown in Fig. 6. To convert

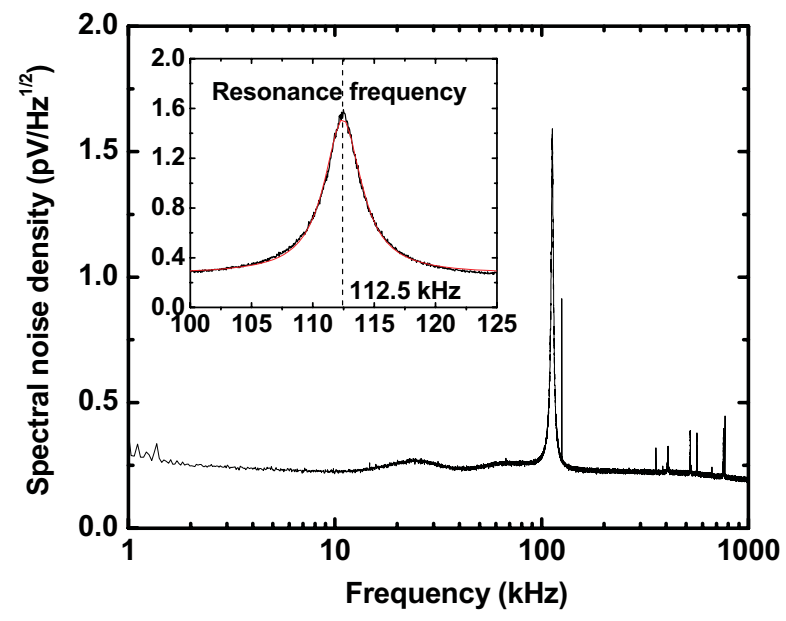

Figure 6. Cantilever mechanical spectral noise density using thermal noise spectrum. The tested cantilever has a resonance frequency of $112.5 \mathrm{kHz}$ as shown in the inset.

a photodiode voltage into an actual displacement, the deflection sensitivity of the photodiode was obtained by taking a forcedisplacement curve on the AFM. By applying equipartition theorem [7] and deflection sensitivity, the spring constant of the cantilever was extracted.
Finite element analysis (FEA) was performed to compare measured mechanical properties with simulation and to investigate high order vibration modes as well. FEMLAB ${ }^{\mathrm{TM}}$ eigen-frequency analysis was used herein. The simulated resonance frequency was $109.2 \mathrm{kHz}$ and showed good agreement with the measurement. FEA results also show several fundamental vibration modes of the ' $U$ '-shaped heated cantilever. High order vibration modes correspond to higher frequency resonance peaks in the thermal noise spectrum (Fig. 6). However, simulated $2^{\text {nd }}$ and $3^{\text {rd }}$ resonant modes were not observed considerably in the thermal noise spectrum partly due to the low sensitivity of photodiode.

Raman spectroscopy is an optical measurement technique well suited for the temperature and stress measurements in microelectromechanical systems (MEMS) devices, especially those made from silicon due to its strong scattering cross-section [8]. Raman Spectroscopy measures the inelastic scattering of light from materials which results from changes in the polarizability of the atoms. Thus, any effect which may change the lattice spacing and polarizability of nonmetallic solids will result in changes in the Raman signature. Raman scattering has been used to determine temperature distribution with micron spatial resolution in crystalline and polycrystalline nonmetallic materials.

Based on our calibration experiments, the position of the Raman Stokes peak is sensitive to both temperature and stress but the line width (FWHM) of the Stokes peak only depends upon the temperature. Differences between the temperatures calculated by the Stokes peak position and line width method should then reveal the effect of stress in devices. Therefore, an analysis of the Stokes spectra will allow the simultaneous determination of temperature and stress.

A novel Raman spectroscopy technique measured local temperature (Fig. 7) and stress (Fig. 8) in a backscattering mode on either side of the cantilever utilizing a specially designed stage that ensured similar conditions for measurements on either side. The cantilever temperature decays roughly exponentially from the tip to the base, indicating a significant amount of heat dissipation into the nearby gas. In addition, the temperature gradient also increases as the excitation voltage increases. The low doped heater shows more significant increases in both temperature and temperature gradient compared to the highly doped legs.

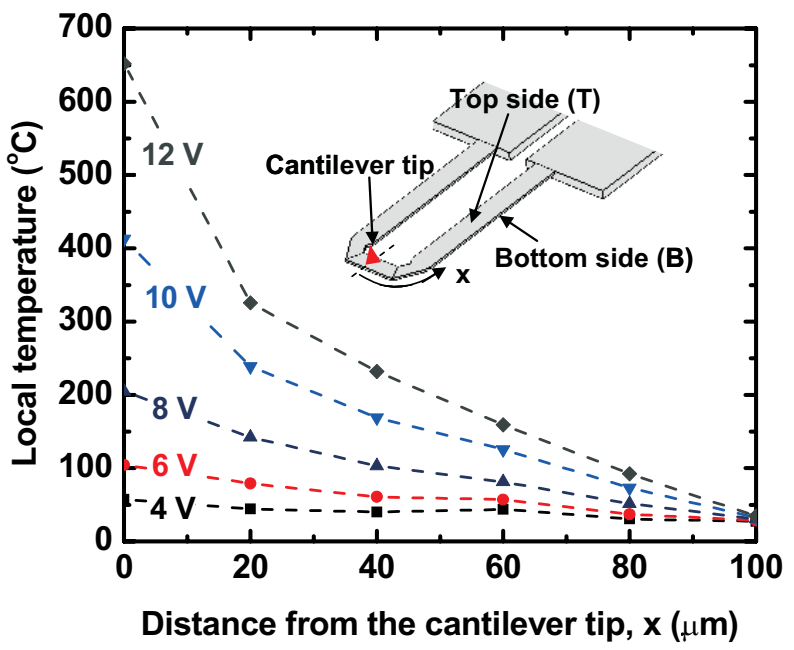

Figure 7. Local temperatures at various locations are measured using Raman spectroscopy. The temperature distribution decays roughly exponentially from the tip to the base, indicating a significant amount of heat dissipation into the nearby gas. 
Figure 8 shows that stress diverges on either side of the cantilever, having elevated stress with increasing temperature on the bottom side and corresponding reduction on the top side. These results confirm that the heated cantilever experiences thermally induced bending which may be caused as a result of the top side doping process. These findings regarding temperature and stress significantly improve upon previously published assumptions regarding the heated cantilever that neglect thermally induced stress and corresponding deflection.
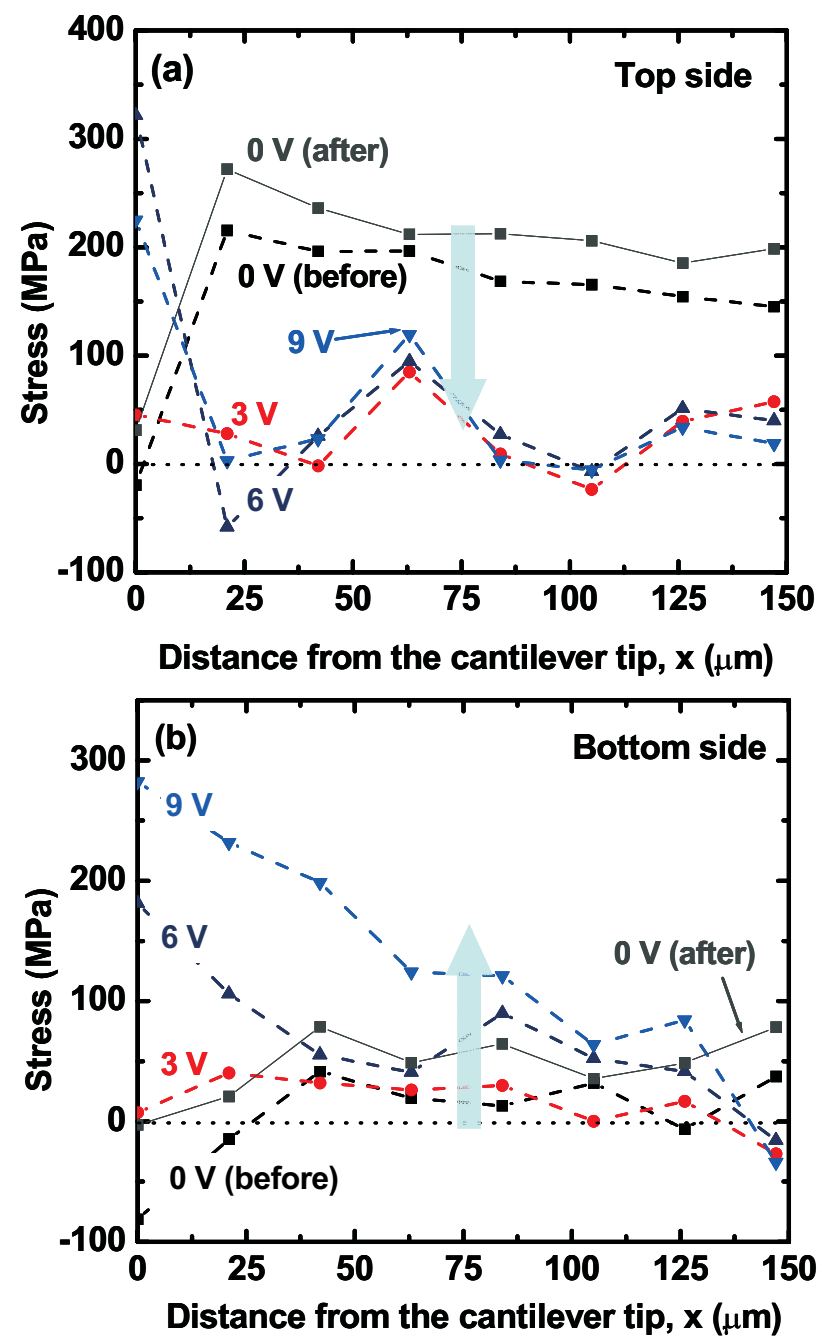

Figure 8. Measured stresses of the heated cantilever using Raman spectroscopy on top and bottom sides. As cantilever power or temperature increases, stress of top side tends to decrease but stress of bottom side seems to increase. The arrows represent changes in stress level as temperature increases. The heated cantilever experiences bending due to nonuniform heating and intrinsic stress. Data near the tip show no trend due to its complex loading state.

\section{CONCLUSIONS}

This paper reports advancements in the characterization and calibration of heated microcantilevers. These measurements help elucidate complex temperature-dependent electrical characteristics of the heated cantilever and suggest how to operate them with DC, pulse, and AC excitation. This first report of frequency-domain operation of heated microcantilevers opens new opportunities for cantilever sensors.
Using Raman spectroscopy, localized temperature and stress are simultaneously examined with $\mu \mathrm{m}$ resolution. Precise temperature calibration was performed with improved precision by $>10 \times$ over previous reports and can extend the use of the heated cantilever beyond data storage. Thermally induced stresses were qualitatively examined from differences in temperature measured by Stokes peak position and line width methods. Mechanical characterization was performed using thermal noise spectrum and mechanical properties such as resonance frequency, Q factor, and spring constant were examined.

This work facilitates new applications of the heated cantilever such as micro/nano calorimeter, mass detection, and nanolithography while a novel laser Raman technique exploited in this work improves design and characterization for other thermal MEMS devices as well.

\section{REFERENCES}

[1] B.W. Chui, T.D. Stowe, Y.S. Ju, K.E. Goodson, T.W. Kenny, H.J. Mamin, B.D. Terris, and R.P. Ried, "Low-stiffness silicon cantilever with integrated heaters and piezoresistive sensors for high-density data storage," Journal of Microelectromechanical Systems, 7, (2002), pp. 69-78.

[2] W.P. King, T.W. Kenny, K.E. Goodson, M. Despont, U. Duerig, M. Lantz, H. Rothuizen, G. Binnig, and P. Vettiger, "Microcantilevers for thermal nanoimaging and thermomechanical surface modification," Technical Digest of the 2002 Solid-State Sensor and Actuator Workshop, Hilton Head, SC (2002).

[3] W.P. King, T.W. Kenny, and K.E. Goodson, "Comparison of thermal and piezoresistive sensing approaches for atomic force microscopy topography measurements," Applied Physics Letters, 85, (2004), pp. 2086-2088.

[4] P.E. Sheehan, L.J. Whitman, W.P. King, and B.A. Nelson, "Nanoscale deposition of solid inks via thermal dip pen nanolithography," Applied Physics Letters, 85, (2004), pp. 15891591.

[5] T. S. Ravi, R. B. Marcus, and D. Liu, "Oxidation sharpening of silicon tips," Journal of Vacuum Science \& Technology, B, 9, (1991), pp. 2733-2737.

[6] B. W. Chui, M. Asheghi, Y. S. Ju, K. E. Goodson, T. W. Kenny, and H. J. Mamin, "Intrinsic-carrier thermal runaway in silicon microcantilevers," Microscale Thermophysical Engineering, 3, (1999), pp. 217-228.

[7] J. L. Hutter and J. Bechhoefer, "Calibration of atomic-force microscope tips," Review of Scientific Instruments, 64, (1993), pp. 1869-1873.

[8] M. R. Abel, T. L. Wright, W. P. King, and S. Graham, "Thermal metrology of silicon micro-structures using Raman spectroscopy," IEEE Transactions Component \& Packaging Techchnology, to be published. 